\title{
Validación de un método de selección para rendimiento en alfalfa basado en la depresión por endocría
}

\author{
Arolfo, V., A. Odorizzi, D. Basigalup y M. Balzarini
}

\begin{abstract}
RESUMEN
La rápida depresión por endocría en alfalfa obedece a la pérdida de interacciones alélicas intra-locus en plantas tri y tetraalélicas. Éstas podrían identificarse por una prueba de autofecundación y luego combinarse en una variedad sintética con mayor rendimiento forrajero. El objetivo de este trabajo fue evaluar la utilidad de la prueba de progenie $\mathrm{S}_{1}$ para identificar genotipos tri y tetraalélicos. Se desarrollaron tres poblaciones sintéticas experimentales (PSE) de alfalfa según tres métodos de selección, partiendo de una población original (PO). El primero, seleccionando las plantas madres que presentaron mayor depresión por endocría $(265 \%)$ en el rendimiento de sus progenies $\mathrm{S}_{1}$; el segundo, seleccionando las plantas de la PO que no formaron semilla $\mathrm{S}_{1}$; el último consistió en la selección fenotípica tradicional de las plantas de la PO con mayores rendimientos (15\% superior). Las seleccionadas fueron polinizadas y cosechadas manualmente, conformando las PSE 1, 2 y 3, respectivamente. Se evaluó la producción de forraje acumulada de cada PSE y PO durante la temporada. Todas las PSE superaron $(p<0,05)$ a la PO, aunque la PSE 1 no se diferenció estadísticamente de la PSE 3. Se estimaron los componentes de la varianza. La heredabilidad $(\mathrm{H})$ alcanzó un valor de 0,86.
\end{abstract}

Palabras clave: Medicago sativa, endocría, mejoramiento, rendimiento de forraje.

Arolfo, V., A. Odorizzi, D. Basigalup and M. Balzarini, 2011. Validation of a selection technique for improving alfalfa forage yield based on inbreeding depression. Agriscientia XXVIII: 13-19

\section{SUMMARY}

Rapid inbreeding depression in alfalfa is due to loss of intraallelic interactions in tri- and tetraallelic plants. These plants could be identified by using a $\mathrm{S}_{1}$ progeny test and then combined into a higher yielding synthetic variety. The objective of this study was to evaluate the usefulness of $S_{1}$ progeny test to identify tri and tetraallelic genotypes. Three alfalfa synthetic experimental populations (PSE) were developed by applying three selection methods to an original plant 
population (PO). The first one, selected those plants whose $\mathrm{S}_{1}$ progenies exhibited higher inbreeding depression ( $265 \%$ ) on forage production; the second one, selected the plants of the PO that did not produce $\mathrm{S}_{1}$ seed; the last one, consisted on traditional phenotypic selection of PO plants with higher forage yield (15\% superior). The elite were manually intercrossed and harvested to produce PSE 1, 2 and 3, respectively. Accumulated forage yield was analyzed for each PSE and PO during the season. All the PSE produced more $(p<0.05)$ than the PO; however, PSE 1 was no different from PSE 3. Data were also used to estimate variance components. The heritability $(\mathrm{H})$ reached a value of 0.86 .

Key words: Medicago sativa, inbreeding, breeding, forage yield.

V. Arolfo, A. Odorizzi, D. Basigalup. EEA Manfredi INTA. Ruta Nac. No 9 km 636, 5988 Manfredi, Córdoba, Argentina. M. Balzarini. Facultad de Ciencias Agropecuarias, Universidad Nacional de Córdoba. CC 509, 5000 Córdoba, Argentina.Correspondencia a: varolfo@manfredi.inta.gov.ar

\section{INTRODUCCIÓN}

Si bien el aumento de la producción de forraje es uno de los objetivos primordiales en todos los programas de mejoramiento genético de la alfalfa (Medicago sativa L.), en las últimas cinco décadas se ha producido sólo un avance limitado en este sentido (Lamb et al., 2006). Por el contrario, la obtención de mayores niveles de resistencia a enfermedades y plagas y la ampliación de la adaptación ambiental han sido exitosas (Hill et al., 1988). Es indudable que el menor progreso en el rendimiento de forraje per se tiene relación directa con la estructura genética de la especie, particularmente con la autotetraploidía y la marcada depresión por endocría, que condicionan los métodos de mejoramiento genético y dificultan la capitalización de la heterosis (Rotilli et al., 1991).

El mejoramiento de la alfalfa se basa casi exclusivamente en la selección fenotípica recurrente, que consiste en la interpolinización de individuos seleccionados para la formación de variedades sintéticas (Rumbaugh et al, 1988). Debido a la naturaleza autotetraploide de la especie, la herencia de los caracteres en alfalfa es compleja. Stanford (1951) fue el primero en determinar la herencia tetrasómica de la especie y en enfatizar la importancia de utilizar dos generaciones para determinarla, dado que el rango total de genotipos esperables después de un cruzamiento sólo se logra luego de dos generaciones de apareamientos al azar. No obstante, en la práctica se considera que el equilibrio genético recién se logra al cabo de cuatro o cinco generaciones en panmixia (Busbice et al., 1972).

Las particularidades tetrasómicas y las interacciones intraalélicas se han postulado como la base primaria de la heterosis en alfalfa, haciendo que su manifestación sea progresiva y que los niveles máximos se alcancen en generaciones avanzadas, aunque dependiendo fuertemente del nivel de endocría de los padres. Según Birchler et al. (2003) en autotretaploides provenientes de dos líneas endocriadas (alelos AABB) existe heterosis, pero es mayor con la presencia de tres o cuatro alelos diferentes en varios loci (ABCD). Los estudios realizados por diversos investigadores (Busbice \& Wilsie, 1966; Bingham et al., 1994) sobre la heterosis progresiva han dado cuenta de que la máxima heterocigosis se asocia con la interacción de múltiples alelos en loci individuales, con segmentos cromosómicos que poseen alelos complementarios, o con una combinación de ambos. Busbice y Wilsie (1966) propusieron que la expresión de la heterosis es máxima para un locus tetraalélico y declina progresivamente para loci tri, di y monoalélicos. Dunbier y Bingham (1975) determinaron que aquellas poblaciones con mayor frecuencia de loci tri y tetraalélicos presentaron los mayores rendimientos de forraje y semilla y los más altos niveles de fertilidad. A pesar de esto, los métodos de mejoramiento genético utilizados en forrajeras limitan el uso de la variación genética no aditiva y consecuentemente la expresión de la heterosis (Hill et al., 1988). La falta de incremento en el ren- 
dimiento de alfalfa puede ser atribuida, en parte, a esta limitante (Brummer, 1999).

Tradicionalmente, la depresión por endocría ha sido considerada opuesta a la heterosis (Wilsie, 1966; Falconer y Mackay, 1986). Aunque las causas de dicha depresión no son completamente conocidas, algunos autores (Lynch \& Walsh, 1998; Willis, 1999) han postulado que -al menos en parte- se debe a la expresión de alelos deletéreos que se van acumulando a medida que la autofecundación fuerza a los loci hacia la homocigosis. La reversión de este proceso permitiría enmascarar estos alelos y restablecer el vigor. Otros autores (Busbice \& Wilsie, 1966; Wilsie, 1966; Salviolli et al., 1976; Bingham et al., 1994) consideran como la causa más importante la pérdida de las interacciones de primer orden en loci tri y tetraalélicos, lo que justificaría la excesivamente rápida depresión por endocría que la alfalfa muestra en la autofecundación. Aún con niveles muy bajos de endocría, la alfalfa manifiesta en la $S_{1}$ una reducción significativa del vigor vegetativo y del rendimiento de forraje y semilla, que se profundiza con sucesivas generaciones de autofecundación (Godeck, 1956; Melton, 1979). Busbice et al. (1972) citan los datos producidos por otros autores en los que el rendimiento de semilla de la $\mathrm{S}_{1}$ fue sólo de $12 \%$ con respecto a la de la generación parental, y en los que la $\mathrm{S}_{8}$ exhibió una reducción de $78 \%$ y $92 \%$ para el rendimiento de forraje y semilla, respectivamente.

En base a lo anterior, y asumiendo que la heterosis en alfalfa está directamente relacionada con el número de interacciones alélicas de primer orden, y que éstas son las responsables del mayor rendimiento forrajero, cabría esperar que aquellas plantas que poseyeran una mayor proporción de loci tri y tetraalélicos deberían mostrar una marcada depresión por endocría en las primeras generaciones de autofecundación, dado que la endogamia llevaría a la pérdida de esas interacciones. De esa forma, la evaluación de la $S_{1}$ permitiría la identificación de los genotipos de mayor valor genético, los que podrían utilizarse luego en la conformación de una población con una significativamente mayor producción de forraje. Por otra parte, y en función de la disminución de la fertilidad provocada por la endocría, también cabría esperar que un número importante de plantas no llegaran a producir semilla $S_{1}$, por la expresión de la pérdida de interacciones intra-locus, además de los mecanismos de auto-incompatibilidad normalmente presentes en la alfalfa. Asumiendo que los individuos que no alcanzaron a formar semilla en la autofecundación fuera debido a la pérdida de las interacciones intralocus podrían ser igualmente valiosos progenitores para la conformación de poblaciones vigorosas y productivas a través de un esquema de policruzas.

En este trabajo se propuso como objetivo evaluar la utilidad de la prueba de progenie $S_{1}$ para la identificación de genotipos portadores de loci tri y tetraalélicos y su posterior combinación para el desarrollo de poblaciones sintéticas.

\section{MATERIALES Y MÉTODOS}

El presente trabajo se desarrolló en el marco del programa de mejoramiento genético de alfalfa del Instituto Nacional de Tecnología Agropecuaria (INTA) y se llevó a cabo en la Estación Experimental Agropecuaria Manfredi (Córdoba, Argentina).

\section{Primera etapa: desarrollo de los sintéticos}

Población original (PO). Se utilizaron 500 plantas de la variedad Bárbara SP INTA, que se produjeron a partir de semillas sembradas, una por maceta, y mantenidas en invernáculo con 12 a 16 h de luz artificial y temperaturas entre 15 y $26^{\circ} \mathrm{C}$, dependiendo de la época del año. Ante la eventualidad de la pérdida de algunas plantas madres (PM) a lo largo del estudio, las 500 plantas fueron clonadas y los clones mantenidos como reserva en condiciones de invernáculo.

Población autofecundada. Cada una de las PM fue autofecundada en forma manual mediante el aplastamiento suave de cada racimo floral con los dedos (Barnes \& Stephenson, 1971). Las vainas, una vez maduras, se cosecharon y trillaron manualmente. De las 500 PM autofecundadas, sólo 264 fueron capaces de producir suficiente semilla para la prueba de progenie de autofecundación, que consistió en sembrar por cada PM dos macetas con tres semillas $\mathrm{S}_{1}$ cada una.

Evaluación de la materia seca (MS). El rendimiento de forraje (g MS planta ${ }^{-1}$ ) de cada PM se registró en cuatro cortes sucesivos. El rendimiento de las progenies $\mathrm{S}_{1}\left(\mathrm{~g}\right.$ MS corte ${ }^{-1}$ ) de cada PM se obtuvo promediando el total de plantas en las dos macetas en cada una de las dos fechas de corte sucesivas que se utilizaron para la evaluación. En todos los casos se cosechó la planta entera (dejando un remanente de $3 \mathrm{~cm}$ ) y los cortes se realizaron cuando la mayoría de las plantas alcanzaba un $10 \%$ de floración o cuando los rebrotes basales medían $5 \mathrm{~cm}$ desde la corona. La determinación de MS se efectuó en estufa a $70^{\circ} \mathrm{C}$ hasta peso constante.

Conformación de las poblaciones sintéticas experimentales (PSE). Se utilizaron tres criterios para conformar las PSE con las PM seleccionadas: i) depresión por endocría, medida en porcentaje de 
reducción en la producción de MS entre las PM y la progenie $S_{1}$; ii) ausencia de formación de semillas después de la autofecundación; y iii) selección fenotípica por mayor producción de forraje (método tradicional). De esta forma se desarrollaron tres sintéticos, conforme al siguiente detalle:

* PSE 1: se policruzaron las 63 PM cuyas progenies $S_{1}$ exhibieron una depresión por endocría (DE) superior al $65 \%$, estimada según la siguiente ecuación:

$$
D E=100-\left(M S S 1 \times M S P M^{-1} \times 100\right)
$$

Las plantas seleccionadas exhibieron valores de DE entre $92,21 \%$ para la planta 3 y $66,83 \%$ para la planta 469.

* PSE 2: de entre las 236 PM que no produjeron semilla por autofecundación, se cruzaron sólo las 138 que exhibieron mayor vigor y mejor aspecto general.

* PSE 3: en base a los datos de producción forrajera de la $\mathrm{PO}$, y a través de un proceso de selección masal tradicional, se identificaron las PM que mostraron los valores más altos de rendimiento de forraje y vigor en los cuatro cortes practicados. Se utilizó una presión de selección de 15\%, a fin de minimizar la consaguinidad en la policruza. En ese contexto, 74 PM presentaron rendimientos de forraje $\geq$ a 23,55 $\mathrm{g} \mathrm{MS}$ planta-1.

Las plantas seleccionadas fueron interpolinizadas manualmente para la conformación de cada uno de los sintéticos. El desenlace floral, con la consecuente carga e intercambio de polen, se realizó mediante una cuña de cartón recortado y plegado en forma de "V". La interpolinización se realizó al azar en el invernáculo con $16 \mathrm{~h}$ de luz y temperaturas de $15 / 26{ }^{\circ} \mathrm{C}$ en la noche y el día, respectivamente. Dentro de cada PSE, la semilla se cosechó en masa y la trilla se realizó manualmente.

El rendimiento forrajero entre las PM y entre las progenies $\mathrm{S}_{1}$ se comparó mediante la prueba $\mathrm{T}$ para observaciones apareadas utilizando el programa Info-Gen (Balzarini y Di Rienzo, 2004), con un nivel de significación del 5\% $(\alpha=0,05)$.

\section{Segunda etapa: evaluación de las PSE}

Ensayo comparativo de rendimiento (ECR). La producción forrajera de los tres sintéticos generados se evaluó en un ECR con un diseño en bloques completos al azar con tres repeticiones y usando como testigo a la PO. Las unidades experimentales consistieron en parcelas de 0,6 $\times 1 \mathrm{~m}$, sembradas con una densidad de $15 \mathrm{~kg} \mathrm{ha}^{-1}$.

El rendimiento de MS se obtuvo cortando y pesando todo el forraje de cada parcela. Los cortes se efectuaron en cuatro fechas sucesivas durante la temporada 2006/07 al estado de 10\% de floración o cuando los rebrotes basales medían aproximadamente $5 \mathrm{~cm}$ desde la corona. La producción

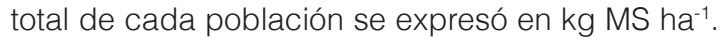
La determinación del porcentaje de MS en cada corte se efectuó sobre el total de forraje producido por cada parcela, secada en estufa a $70{ }^{\circ} \mathrm{C}$ hasta peso constante.

Análisis estadístico. Con la información producida en cada corte se calculó la producción acumulada de forraje a lo largo de la temporada, a la que se le realizó un análisis de la varianza. Las medias se compararon a través de la prueba de comparaciones múltiples $\operatorname{LSD}(\alpha=0,05)$ con el paquete estadístico Info-Gen (Balzarini y Di Rienzo, 2004).

A fin de estimar la heredabilidad en sentido amplio $(\mathrm{H})$ del carácter rendimiento de forraje, se estimaron los componentes de la varianza mediante la técnica de máxima verosimilitud residual (restricted maximum likelihood) (Fry, 2003) usando el paquete estadístico S.A.S a partir de PO y las PSE del ECR. De esta forma, se obtuvieron los valores de la varianza poblacional $\left(\sigma_{\mathrm{P}}^{2}\right)$ y de la varianza residual $\left(\sigma_{E}^{2}\right)$, y a partir de ellos se calculó la varianza fenotípica $\left(\sigma_{F}^{2}=\sigma_{P}^{2}+\sigma_{E}^{2}\right)$ y se estimó la $H$ en función de la siguiente ecuación:

$$
H=\left(\sigma_{p}^{2}\right) /\left(\sigma_{F}^{2}\right)
$$

\section{RESULTADOS Y DISCUSIÓN}

\section{Primera etapa: desarrollo de los sintéticos}

Material de autofecundación. Se registró una gran diferencia entre las plantas de la PO respecto de su capacidad relativa para producir semillas luego de la autofecundación. En ese contexto, y con el objeto de simplificar el manejo, las plantas se clasificaron en "no productoras" y "productoras" de semilla $S_{1}$. Dentro del primer grupo, se observó caída y aborto de flores. Dentro del segundo grupo, se obtuvieron frutos tanto normales como anormales, con pocas espiras y de una a tres semillas por vaina. Esto último es coincidente con lo obtenido por otros autores (Heinrichs, 1968; Barnes et al., 1972; Marble et al., 1986; Viands et al., 1988), quienes informaron sobre una marcada disminución de la producción de semillas por planta después de la autofecundación. Como ya fuera mencionado anteriormente, esta caída en la fertilidad puede ser la consecuencia de los mecanismos de autoicompatibilidad $\mathrm{y} / \mathrm{o}$ autoesterilidad presentes en alfalfa y/o de la pérdida de las interacciones intra-locus.

Evaluación de la población original. Los valores de rendimiento acumulado en los cuatro cortes de forraje efectuados en la PO resultaron extremada- 
mente variables, con un rango que fue desde los 38,29 g MS hasta los 4,75 g MS. Los valores más frecuentes se ubicaron entre los 13 y los $21 \mathrm{~g} \mathrm{MS}$ planta $^{-1}$.

Evaluación de las progenies $S_{1}$. El rendimiento de forraje acumulado promedio de los dos cortes del material $\mathrm{S}_{1}$ también resultó extremadamente variable y fue desde los $21,77 \mathrm{~g}$ MS a los $0,80 \mathrm{~g}$ MS.

Del análisis a través de la prueba T para observaciones apareadas se desprende que las diferencias entre la PO y las progenies $S_{1}$ fueron significativas (Tabla 1). En la Figura 1 se aprecia que, en general, las progenies autofecundadas produjeron menos forraje que sus plantas madres, lo que evidencia una depresión por endocría. Esto último, al igual que lo señalado para la producción de semilla, es coincidente con lo señalado por Busbice y Wilsie (1966), Wilsie (1966), Desereaux y Gallais (1969), Salvioli et al. (1976) y Bingham et al. (1994).

\section{Segunda etapa: evaluación de las poblaciones}

Ensayo comparativo de rendimiento. El análisis

Tabla 1. Prueba $T$ para muestras apareadas para la media del rendimiento de forraje de alfalfa de las plantas madres vs. media de las progenies $\mathrm{S}_{1}$.

\begin{tabular}{ccccccc}
\hline Obs 1 & Obs 2 & $\mathrm{N}$ & $\chi$ & $\mathrm{DE}($ dif $)$ & $\mathrm{T}$ & Bilateral \\
\hline $\mathrm{PO}$ & $\mathrm{S}_{1}$ & 252 & 1,85 & 2,24 & 13,09 & $<0,0001$ \\
\hline
\end{tabular}

PO: población original; $\mathrm{S}_{1}$ : progenie autofecundada; $\chi$ : media; $\mathrm{DE}$ (dif): desviación estándar de la diferencia

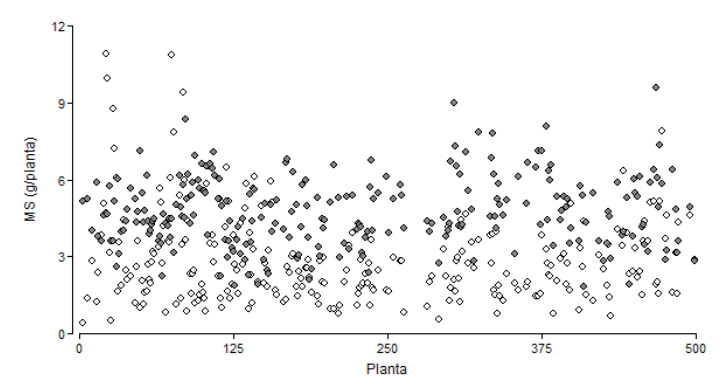

Figura 1: Diagrama de dispersión de los valores promedio por planta de alfalfa de la producción de forraje de la población original y su descendencia $S_{1}$ medidos en $\mathrm{g} \mathrm{MS}$ planta $^{-1}$ donde: $O$ plantas madres y $O$ progenies $\mathrm{S}_{1}$. de la varianza de la producción de forraje acumulada (cuatro cortes) arrojó diferencias significativas $(p<0,05)$ entre la PO y las tres PSE desarrolladas (Tabla 2), lo que indica la efectividad de cualesquiera de los tres criterios de selección empleados en este trabajo para la conformación de los sintéticos experimentales. Entre estos últimos, la PSE 3 (selección masal) fue la de mayor rendimiento, con un valor de 14.576,37 $\mathrm{kg} \mathrm{MS} \mathrm{ha-1}^{-1}$, mientras que la PSE 2 (policruza de las plantas más vigorosas que no produjeron semilla $S_{1}$ ) fue la de menor rendimiento, con un valor de 12.382,47 $\mathrm{kg} \mathrm{MS} \mathrm{ha}^{-1} \mathrm{y}$ con diferencias significativas con la primera. Por su parte, la PSE 1 (selección de PM basada en la depresión por endocría) se ubicó en una posición intermedia y no presentó diferencias con ninguna de las otras dos poblaciones (Figura 2).

En función de todo lo anterior, se infiere que para aumentar el rendimiento de forraje per se, el método de selección basado en la depresión por endocría -estimada a través de una prueba de progenie $\mathrm{S}_{1}$ - fue tan efectivo como la selección masal. Sin embargo, en términos de practicidad y eficiencia, pareciera que el mayor esfuerzo que demanda la inclusión de una prueba de autofecundaciones no justificaría su empleo en un programa de desarrollo

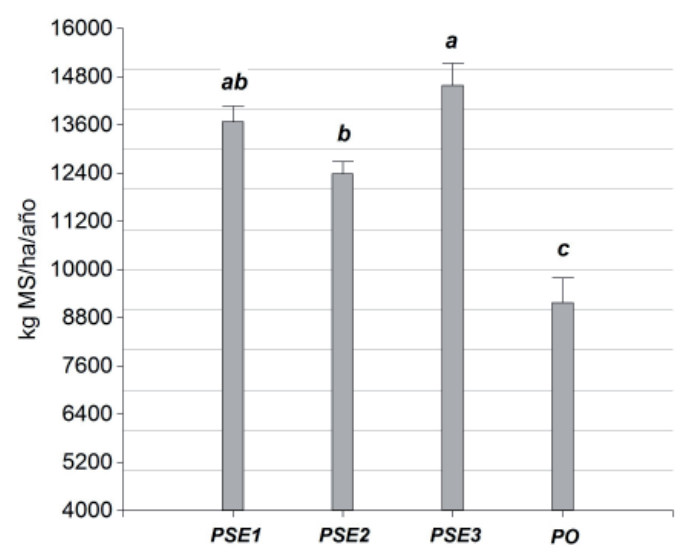

Figura 2: Comparación del rendimiento de forraje en materia seca acumulado ( $\mathrm{kg} \mathrm{MS} \mathrm{ha-1}$ año-1) entre los sintéticos desarrollados, donde PSE 1: mayor depresión por endocría; PSE 2: ausencia de semillas en la autofecundación; PSE 3: mayor rendimiento de forraje; PO: población original. Barras con la misma letra no difieren estadísticamente $(p>0,05)$.

Tabla 2. Resumen del análisis de la varianza para la variable rendimiento de forraje de alfalfa de las PSE y de la PO.

\begin{tabular}{lccccc}
\hline Fuente de variación & Suma de cuadrados & GL & Cuadrados medios & Valor de F & Pr $>$ F \\
\hline Modelo & 51032173,45 & 5 & 10206434,69 & 12,21 & 0,0042 \\
Poblaciones & 50357358,28 & 3 & 16785786,09 & 20,09 & 0,0016 \\
Repeticiones & 674815,17 & 2 & 337407,59 & 0,40 & 0,6847 \\
Error & 5013856,86 & 6 & 835642,81 & \\
\hline
\end{tabular}


de variedades. No obstante, es necesario tener en cuenta que en este estudio se compararon sintéticos que estaban en su primera generación de síntesis (Syn 1) y que, por lo tanto, no han alcanzado todavía su equilibrio genético. En consecuencia, a fin de extraer conclusiones más robustas, habría que comparar estos mismos sintéticos en generaciones más avanzadas (Syn 3 o Syn 4), cercanas al equilibrio genético. De esa forma, la comparación de poblaciones más estables podría dar una estimación más certera de la verdadera validez de cada método de selección.

La heredabilidad en sentido amplio $(\mathrm{H})$ del carácter rendimiento de forraje se estimó en 0,86 (Tabla 3). Este valor alto indica que, al menos para las condiciones en que se llevó a cabo este estudio, el mayor efecto dentro de la varianza fenotípica fue producido por los genotipos, lo que se vio reflejado en la significativa respuesta a la selección que se observó con todos los métodos de mejoramiento empleados.

Tabla 3. Resumen del análisis de los componentes de la varianza y valor de heredabilidad para el rendimiento de forraje, donde $\sigma_{\mathrm{p}}^{2}$ : varianza poblacional; $\sigma_{E}^{2}$ : varianza residual; $\mathrm{H}$ : heredabilidad.

\begin{tabular}{lc}
\hline Componentes de la varianza & Valor \\
$\sigma^{2} \mathrm{p}$ & 5316714 \\
$\sigma^{2} \mathrm{E}$ & 835643 \\
$\mathrm{H}$ & 0,8641 \\
\hline
\end{tabular}

\section{CONCLUSIONES}

Tanto la selección masal como la basada en la depresión por endocría de las progenies $\mathrm{S}_{1}$, resultaron efectivas $(p<0,05)$ para mejorar el rendimiento de forraje en las tres PSE (Syn 1) desarrolladas respecto de la población original de la cual derivaron. Sin embargo, la selección basada en la depresión por endocría de la producción de MS no probó ser superior a la selección fenotípica tradicional basada en la identificación de las plantas madres más rendidoras. De todos modos, sería necesario establecer la comparación entre los sintéticos sobre la base de generaciones de síntesis más cercanas al equilibrio genético (Syn 3 o Syn 4).

\section{BIBLIOGRAFÍA}

Balzarini M. y J. Di Rienzo, 2004. InfoGen: Software estadístico para el análisis de datos genéticos. Universidad Nacional de Córdoba, Argentina. www.Info-Gen. com.ar.

Barnes, D.K.; E.T. Bingham, J.D. Axtell and W.H. Davis, 1972. The flower, sterility mechanisms and pollination control. In: Hanson, C.H. (Ed). Alfalfa Science and Technology ASA-CSSA-SSSA. Madison, WI, USA. Agronomy Series N 15, pp. 123-141.
Barnes, D.K. and M.G. Stephenson, 1971. Relative Efficiencies of Four Self-Pollination Techniques in Alfalfa. Crop Sci. 11:131-132.

Bingham, E.T.; R.W. Groose, D.R. Woodfield and K.K. Kidwell, 1994. Complementary gene interactions in alfalfa are greater in autotetraploids than diploids. Crop Sci. 34:823-829.

Birchler, J.A.; D.L. Auger and N.C. Riddle, 2003. In search of the molecular basis in heterosis. The Plant Cell, Vol. 15, 2236-2239.

Brummer, E.C., 1999. Capturing heterosis in forage crop cultivar development. Crop Sci. 39:943-954.

Busbice, T.H. and C.P. Wilsie, 1966. Inbreeding depression and heterosis in autotetraploids with application to Medicago sativa L. Euphytica 15:52-67.

Busbice, T.H.; R.R. Hill and H.L. Carnahan, 1972. Genetics and Breeding Procedures. In: Hanson, C.H. (Ed). Alfalfa Science and Technology. ASA-CSSA-SSSA. Madison, WI, USA. Agronomy Series No 15, pp. 283318.

Desereaux, L. and A. Gallais, 1969. Inbreeding and heterosis in autotetraploid alfalfa. I Fertility. Can. J. of Genetics and Cytology. Vol. XI, Number 3:706-715.

Dunbier, M.W. and E.T. Bingham, 1975. Maximum heterozygosity in alfalfa: results haploid derived autotetraploid. Crop Science 14:815-819.

Falconer, D.S. y T.F.C. Mackay, 1986. Introducción a la genética cuantitativa. Editorial Acribia. Zaragoza (España). 468 pp.

Fry, J.D., 2003. Estimation of genetic variances and covariances by restricted maximum likelihood using PROC MIXED. In Saxton A.M. (Ed). Genetic analysis of complex traits using SAS. SAS Institute, Cary, NC. pp. 11-34.

Godeck, W., 1956. Información preliminar sobre autofecundación en alfalfa. IDIA. INTA Buenos Aires (Argentina) 98:27-29.

Heinrichs, D.H., 1968. Alfalfa in Canada. Canada Department of Agriculture. Pub. 1377. pp. 5-9.

Hill, R.R. (Jr); J.S. Shenk and R.F. Barnes, 1988. Breeding for yield and quality. In: Hanson, A.A.; D.K. Barnes and R.R. Hill (Jr) (Ed). Alfalfa and Alfalfa Improvement. ASA-CSSA-SSSA. Madison, WI, USA. Agronomy. Series $N^{\circ} 29$, pp. 809-825.

Lamb, J.F.S; C.C. Sheaffer, L.H. Rhodes, R.M. Sule, D.J. Undersander and E.C. Brummer, 2006. Five decades of alfalfa cultivar improvement: impact on forage yield, persistence and nutritive value. Crop Sci. 46:902-909.

Lynch, M. and B. Walsh, 1998. Genetics and analysis of quantitative traits. Sinauer Associates, Sunderland, MA, USA, 980 pp.

Marble, V.L.; L.H. Ochoa y C.J. Moschetti, 1986. Producción de semilla de alfalfa. En: Bariggi, C.; C.D. Itria; V.L. Marble y J.M. Brun (Ed.). Investigación, producción y tecnología de alfalfa. Buenos Aires, Arg. Colec- 
ción Científica del INTA. Tomo XXII, pp. 371-442.

Melton, B., 1979. Inbreeding, environmental effects and combining ability studies in alfalfa. New Mexico State Univ. Agr. Exp. Stn. Special Report 35, 223 pp.

Rotilli, P.; G. Gnocchi, C. Scotti and L. Zannone, 1991. Some aspects of breeding methodology in alfalfa. http://www.naaic.org/TAG/TAGpapers/rotili/rotili.html [consultado 05/09/2005].

Rumbaugh, M.D.; J.L. Caddel and D.E. Rowe, 1988. Breeding and Quantitative Genetics. In: Hanson, A.A.; D.K. Barnes and R.R. Hill (Jr) (Ed). Alfalfa and Alfalfa Improvement. ASA-CSSA-SSSA. Madison, WI, USA. Agronomy. Series No 29, pp. 777-808.

Salvioli, R.A.; L.A. Suárez, M.L. Collado, C.A. Latina y G.O. Martin, 1976. La selección en alfalfa (Medicago sativa L.). Rev. Agron. N.O. Argent. XIII (1-4), pp. $87-$ 104.
Stanford,,E.H., 1951. Tetrasomic inheritance in alfalfa. Agr. Jour. 43:5, 222-225.

Viands, D.R.; P. Sun and D.K. Barnes, 1988. Pollination control: mechanical and sterelity. In: Hanson, A.A.; D.K. Barnes and R.R. Hill (Jr) (Ed.). Alfalfa and Alfalfa Improvement. ASA-CSSA-SSSA. Madison, WI, USA. Agronomy Series N²9, pp. 931-960.

Willis, J.H., 1999. Inbreeding load, average dominance and the mutation rate for mildly deleterious alleles in Mimullus guttatus. Genetics 153:1885-1898.

Wilsie, C.P., 1966. Inbreeding depression and heterosis in Medicago sativa, selfing and slower forms of inbreeding. Jour. Paper No J-5241 lowa Agr. And Home Econom. Exp. Stn, Ames, lowa. Project No 1048. (reprinted from Proceedings of the $X$ International Grassland Congress, 1966). 